\title{
Study and Development of Multi Pixel Photon Counter for the GLD Calorimeter Readout
}

\author{
Satoru Uozumi* \\ Faculty of Science, Shinshu University \\ E-mail: satorueazusa.shinshu-u.ac.jp \\ for the GLD Calorimeter Group
}

The Multi Pixel Photon Counter (MPPC) is a novel photon sensor manufactured by Hamamatsu Photonics. It has many advantages compared to other photon sensors (compact size, low cost, high gain and detection efficiency, insensitivity to magnetic fields). In the GLD calorimeter group, study of the MPPC properties is extensively ongoing aiming to utilize it as the photon sensor of a finely segmented calorimeter. As a result of the study, we have confirmed that the current 1600 pixel MPPC has almost satisfactory gain, photon detection efficiency and noise rate for our requirements. We have also found that the dynamic range of the 1600 pixel MPPC can be enhanced thanks to its short recovery time, which is useful for calorimetric use. As a practical test for use at the GLD calorimeter, we have constructed a prototype of a scintillator-strip calorimeter and exposed it to 1-6 GeV positron beams and validated its performance. As a result of the test, we have observed a satisfactory linearity and energy resolution of the scintillator-strip calorimeter with full MPPC readout.

International workshop on new photon-detectors PD07

June 27-29 2007

Kobe University, Kobe, Japan

\footnotetext{
* Speaker.
} 


\section{Introduction}

The International Linear Collider (ILC) is a next generation $e^{+} e^{-}$collider with a center-ofmass energy up to $1 \mathrm{TeV}$. The high energy $e^{+} e^{-}$collisions provide us with an ideal environment, not only to study properties of standard model particles, but also to explore several possibilities of new physics beyond the standard model. The Global Large Detector (GLD) is one of the four detector concepts which have been proposed for the ILC experiment [1]. At the ILC experiment, since many important physics processes will emerge as multi-jet final states, precise jet energy measurement is one of the most crucial issues. Practically the goal of the jet energy resolution is set to $\sigma_{E} / E=$ $30 \% / \sqrt{E}$, where $E$ and $\sigma_{E}$ denote the jet energy and the uncertainty of its measurement. To achieve such a high precision measurement, a powerful method, called Particle Flow Algorithm(PFA), is adopted for jet-energy reconstruction at GLD [2]. PFA takes advantage of the better resolution of momentum measurement in the central tracker than energy resolution of the calorimeter. In the PFA particles in a jet are categorized, and their momenta or energy are measured as follows:

- Momenta of charged particles ( $\sim 65 \%$ in a jet) are measured by central tracker,

- Energy of photons (mainly from $\pi^{0}$ decays, $\sim 25 \%$ in a jet) are measured by electromagnetic calorimeter,

- Energy of neutral hadrons (mainly $K_{L}^{0}, \sim 10 \%$ in a jet) are measured by hadron calorimeter.

To use this method, separation of the jet particles in the calorimeter is indispensable to avoid miscounting of the energy. This requirement results in a requirement of the finely segmented calorimeter, with a cell size of order $1 \times 1 \mathrm{~cm}$. The GLD calorimeter is a sandwich calorimeter which consists of absorber (tungsten for electromagnetic, lead or iron for hadronic part) and scintillator layers, placed inside 3 Tesla magnetic field. As shown in Figure 1, the scintillator layer is constructed of scintillator strips whose size is $4.5 \times 1 \mathrm{~cm}$ with a thickness of $3 \mathrm{~mm}$. In successive scintillator layers, strips are alternately aligned in orthogonal directions to achieve the fine segmentation. Since the number of scintillator strips is huge ( $\sim 14$ million) and all the signals have to be read out individually, the readout photon sensor is a key issue for the GLD calorimeter. The Multi-Pixel Photon Counter (MPPC) is a powerful candidate which has many attractive features (compact size, low cost, good performance, and magnetic-field tolerance and so on) and is therefore feasible for the GLD calorimeter readout [B] [4][5]. However since the MPPC is a novel, still evolving device, we are studying its properties in collaboration with Hamamatsu Photonics, to improve its performance and eventually use it in the GLD calorimeter.

\section{Performance of the 1600 pixel MPPC}

We have been studying the performance of the small-package 1600 pixel MPPC (Figure 凤) which is suitable to attach to the scintillator strip. At first, we have set practical requirements of the MPPC performance for the GLD calorimeter as follows:

- Gain and photon detection efficiency are comparable to conventional photomultipliers. 


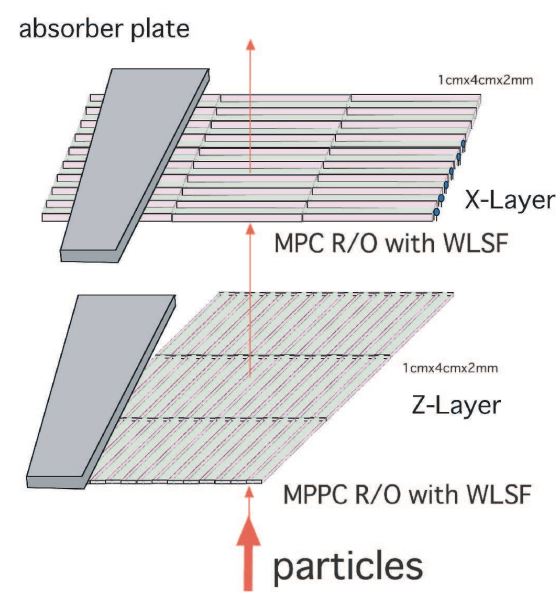

Figure 1: A schematic view of the GLD scintillator-strip calorimeter.

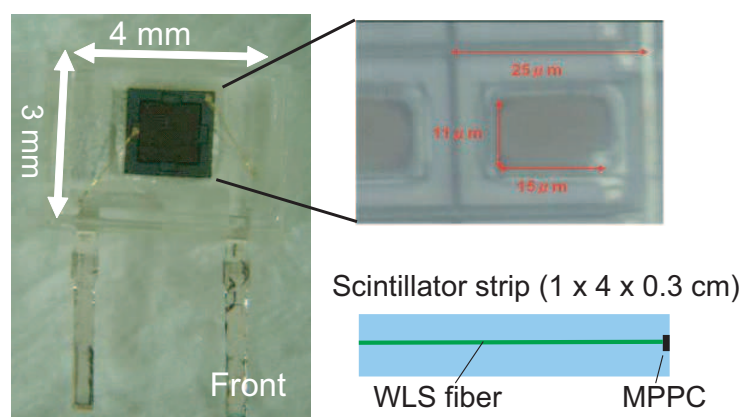

Figure 2: The small-package 1600 pixel MPPC and its microscopic view. This small MPPC is attached to the scintillator strip as shown in figure.

- Low dark noise rate $(\sim 1 \mathrm{MHz})$, and inter-pixel cross-talk probability (a few \%).

- Dynamic range is enough to measure up to $100 \mathrm{GeV}$ photons.

- Stable for long-term use and robust against radiation and magnetic fields.

- Uniform performance among many pixels.

- Low cost, compactness.

To achieve these goals, extensive R\&D has already been performed. Here we show the performance of the latest 1600 pixel MPPC.

Results of the gain measurement are shown in Figure 3. The gain has a linear relation with over-voltage $\Delta V=V_{\text {bias }}-V_{0}$ and expressed as,

$$
\text { Gain }=\frac{C}{e}\left(V_{\text {bias }}-V_{0}\right)=\frac{C}{e} \Delta V
$$

where $C$ and $V_{0}$ denote the pixel capacitance and breakdown voltage, $e$ is charge of the electron. From the result of the measurement, the gain of the 1600 pixel MPPC is more than $10^{5}$, which is satisfactory for our requirements. It is also found that the breakdown voltage has a temperature coefficient of $\Delta V_{0} / \Delta T=56.0 \pm 0.1 \mathrm{mV} / \mathrm{C}^{o}$.

The dark noise caused by thermions is measured and displayed in Figure 4 . It significantly depends on temperature, however even at room temperature $\left(\sim 25 \mathrm{C}^{o}\right)$ the typical rate is order of $100 \mathrm{kHz}$ which is sufficiently small. The probability of inter-pixel cross-talk is measured assuming that dark noise with more than 1 pixel fired dominantly comes from dark noise together with crosstalk to nearby pixels:

$$
P_{\text {cross-talk }}=\frac{\text { Rate of } \geq 1 \text { pixel fired noise }}{\text { Rate of } \geq 2 \text { pixel fired noise }}
$$



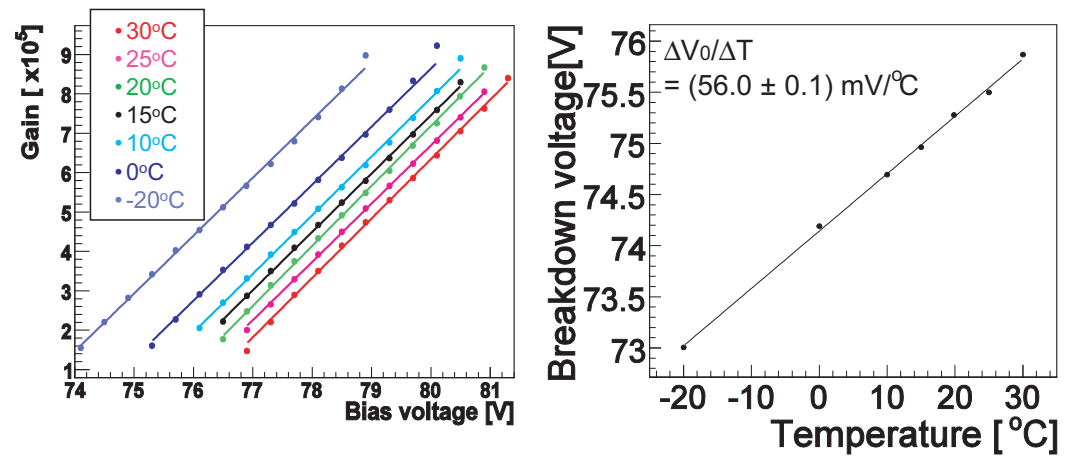

Figure 3: The measured gain as a function of bias voltage (left), and temperature dependence of the breakdown voltage (right).

The cross-talk probability increases with over-voltage, however does not strongly depend on temperature.
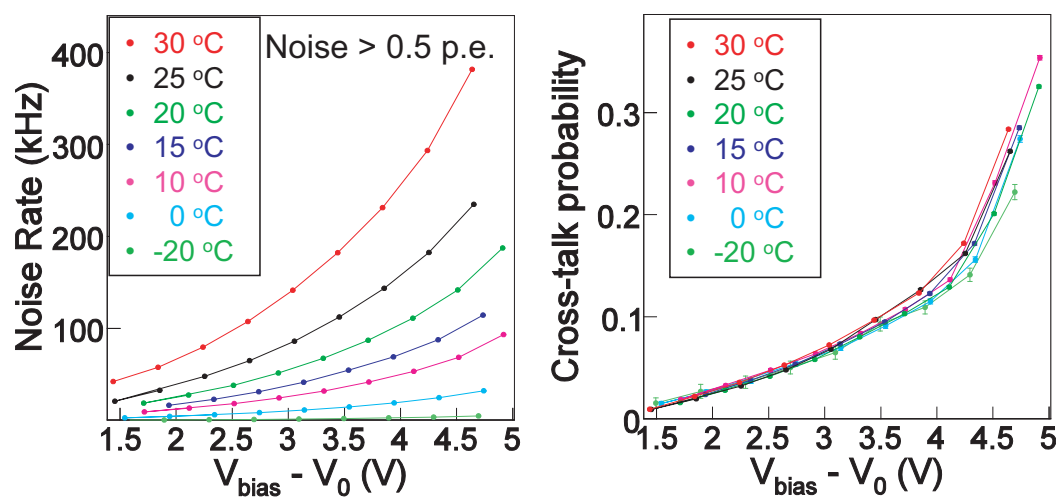

Figure 4: The dark noise rate and the probability of inter-pixel cross-talk, as a function of over-voltage. Results at different temperatures are overlaid.

We have also measured the variation of pixel capacitance, breakdown voltage and dark noise rate over 700 samples of the 1600 pixel MPPCs as shown in Figure 5. The variation is less than $4 \%$ for $C$ and $V_{0}$, and of order $10 \%$ for the dark noise rate, which are acceptably small. Overall these basic properties of the latest 1600 pixel MPPC are almost satisfactory for the requirement of the GLD calorimeter.

Then we have measured the photon detection efficiency (PDE) of the 1600 pixel MPPC with the setup shown in Figure 6. Light pulses from a LED are guided by a wavelength shifting fiber (WLSF) and fed into the MPPC or photomultiplier through a $1 \mathrm{~mm}$ diameter pin-hole. The photon detection efficiency of the photomultiplier is known (16\%). By injecting the same light pulse into both the MPPC and photomultiplier and comparing the observed light yields from both sensors, one can extract the PDE of the MPPC as

$$
\mathrm{PDE}(\mathrm{MPPC})=\frac{N_{\text {p.e. }}(\mathrm{MPPC})}{N_{\text {p.e. }}(\mathrm{PMT})} \mathrm{PDE}(\mathrm{PMT}) .
$$



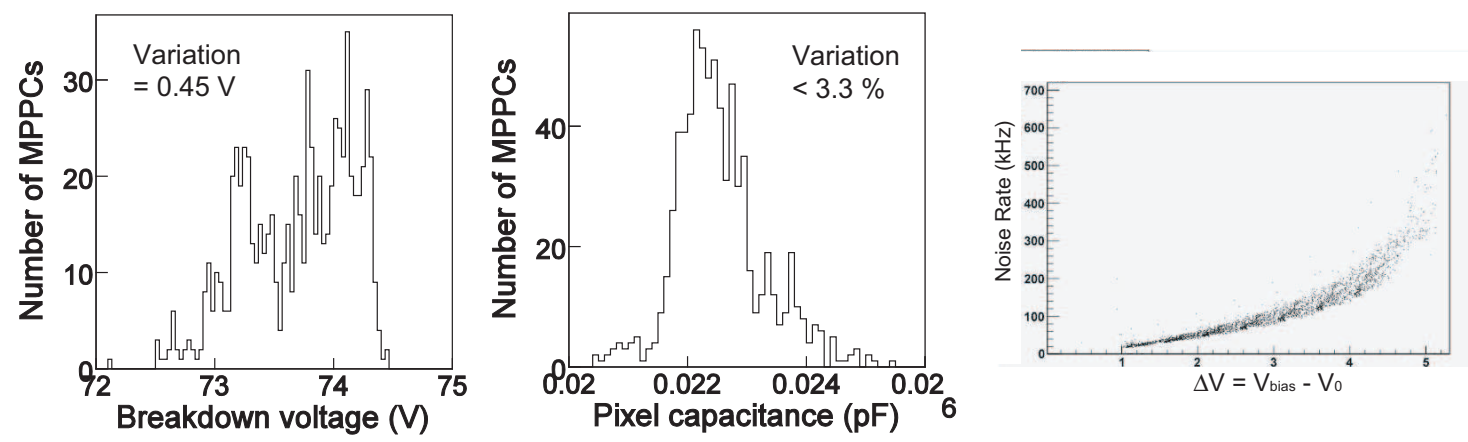

Figure 5: Variation of the breakdown voltage (left), pixel capacitance (middle), and dark noise rate (right) over 700 samples.

The measured PDE is shown in Figure 6, as a function of the over-voltage. The PDE is saturated at $\Delta V>3 \sim 4 \mathrm{~V}$, with a maximum value is around $17 \%$, comparable to conventional photomultipliers.
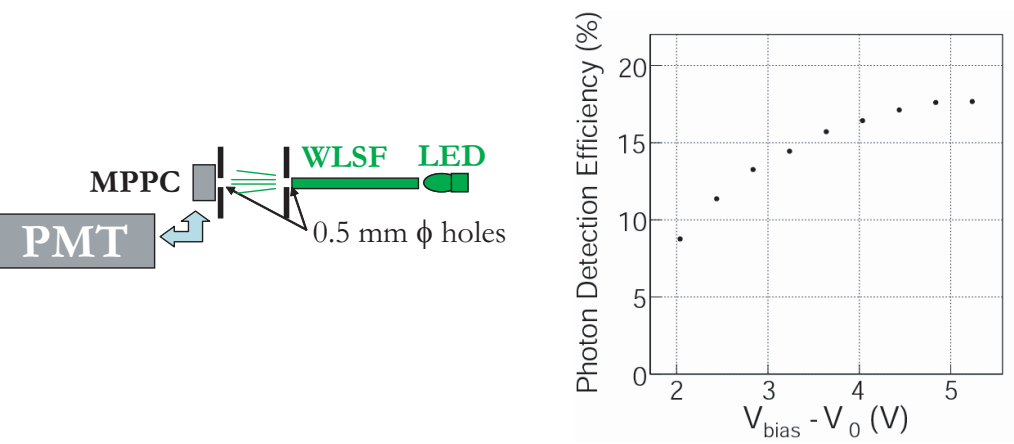

Figure 6: Setup and result of the PDE measurement.

Another important issue for the calorimetric use of the MPPC is its dynamic range and response to strong light ( $\sim$ several thousands photons). The MPPC has a fundamentally non-linear response to light input, since it has a finite number of pixels. If the light input comes within very short time ( $\sim 100 \mathrm{ps})$, the output of the 1600 pixel MPPC can be described as

$$
N_{\text {fired }}=N_{\text {pix }}\left(1-e^{-N_{\text {p.e. }} / N_{\text {pix }}}\right),
$$

where $N_{\text {pix }}=1600$ pixels, and $N_{\text {p.e. }}=N_{\text {photons }} \cdot \mathrm{PDE}$ is the number of photoelectrons detected by a sensor. However if the recovery time of a pixel is short enough compared with the length of the input light pulse, a pixel can recover after firing the first avalanche signal and may fire several times. Since the recovery time of the 1600 pixel MPPC is relatively short ( $\sim 4 \mathrm{~ns}$, as described later), the dynamic range may be enhanced with the light signal from the scintillator strip and the WLSF. To explore this effect, we have measured the recovery time of the 1600 pixel MPPC. The setup is shown in Figure 7. At first a short bright laser light pulse is injected into the MPPC to make all the pixels fire. Then after $\Delta t$ of delay, another bright LED light pulse is injected into the MPPC. If $\Delta t$ is large enough, the MPPC gives a signal corresponding to 1600 avalanches. However if $\Delta t$ is short 
and the second light pulse comes during the pixel recovery phase, the pulse height of the MPPC output becomes lower than the former case. By measuring the MPPC output to the second pulse at various $\Delta t$, one can observe the recovery curve and its time constant. The observed recovery curve as a function of $\Delta t$ is shown in Figure 7). The plot shows the recovery fraction, which means the fraction of gain recovery. By fitting the result with an empirical function $f=A\left(1-e^{-(\Delta t-a) / \tau_{r}}\right)$, the recovery time $\tau_{r}$ is extracted to be $4.1 \pm 0.1 \mathrm{~ns}$ at $V_{\text {bias }}=71.0 \mathrm{~V}$. We have also measured the curve at different bias voltages, however its effect on the recovery curve is negligible.
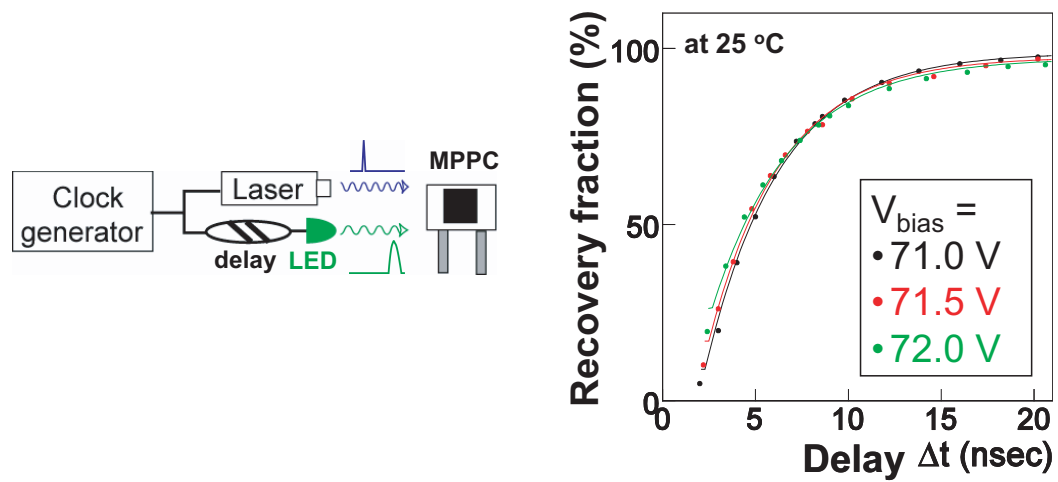

Figure 7: Setup and and result of the recovery time measurement.

Then we measure the actual response curve of the 1600 pixel MPPC. As previously discussed, if the length of the input light is close to or longer than the recovery time ( $\sim 4 \mathrm{~ns})$, the effective number of pixels can be increased and eventually the dynamic range for such signals becomes wider. Therefore we have measured saturation curves with different widths of input light pulses. As shown in Figure 8, a rectangular pulse with width $w$ ns is fed into the LED, and the LED light pulse is injected into the MPPC. The observed response curves with different values of $w$ are also shown in Figure 8. The true number of photons injected (corresponding to the horizontal axis) is measured using a photomultiplier. One can see that the response curves show different shapes with different values of $w$. The response curve with larger $w$ shows wider dynamic range as expected. We also change the bias voltages, however this does not give any significant effects to the response curve. This effect, enhancement of the dynamic range by the short recovery time, would be useful for the calorimetric use of the MPPC where a larger dynamic range is important. However, to take advantage of this effect, the shape of the light signal from the scintillator strip should be precisely determined.

\section{GLD Electromagnetic Calorimeter Prototype}

As a practical test of the construction of the scintillator-strip calorimeter with MPPC readout, we have constructed an electromagnetic calorimeter prototype and exposed it to $1-6 \mathrm{GeV}$ positron beams to evaluate its performance [6].

The structure of the test module is shown in Figure 9. The module consists of 26 pairs of scintillator and tungsten layers of thickness 3 and $3.5 \mathrm{~mm}$ respectively. The scintillator layer has 

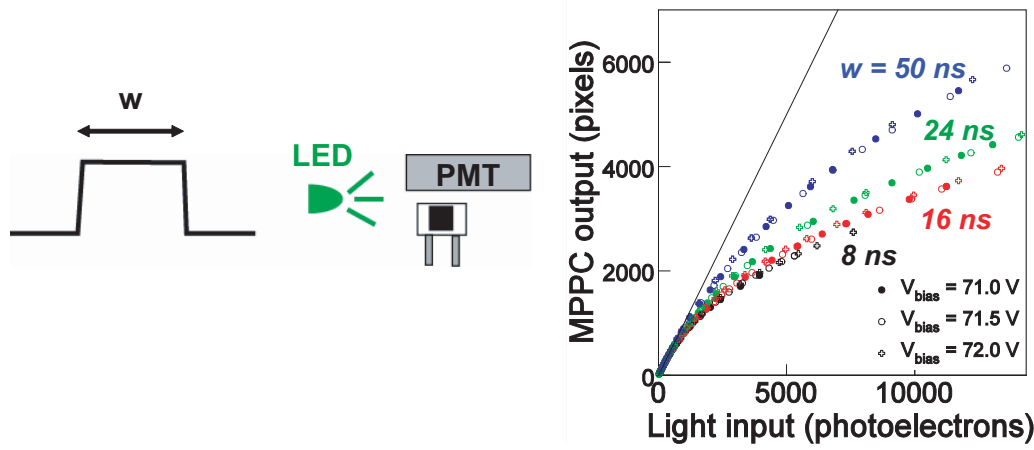

Figure 8: Setup of the response curve measurement (left) and response curves of the 1600 pixel MPPC measured with different length of light pulse injected (right).

$9 \times 2$ scintillator strips of size $4.5 \times 1 \mathrm{~cm}$. In successive scintillator layers, the strips are alternately aligned vertically and horizontally. The 1600 pixel MPPC is mounted at the edge of each scintillator strip. The light signal from the scintillator is guided through a WLSF and read by the MPPC. The size of the entire module is about $9 \times 9 \times 20 \mathrm{~cm}$ and the total number of readout channels is 468 .

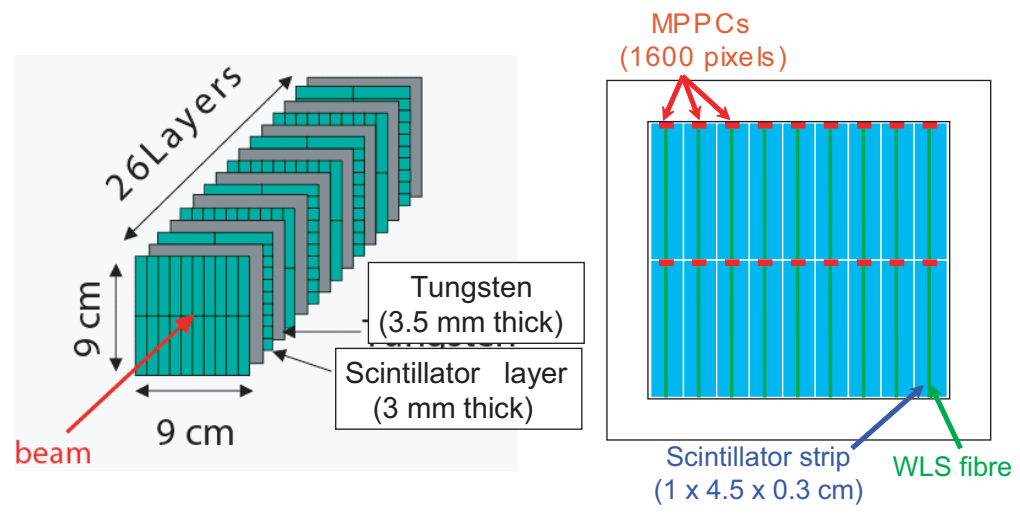

Figure 9: The structure of the GLD electromagnetic calorimeter test module.

A beam test was performed at the DESY-II electron synchrotron in March 2007 using 1-6 GeV positron beams. At each energy, positrons with well-determined momenta were injected into the center of the test module, and the energy resolution and linearity of the test module were evaluated. Results of the beam tests are shown in Figure 10. The energy resolution for the positron is measured to be $\sigma / E=(13.45 \pm 0.07) / \sqrt{E} \oplus(2.87 \pm 0.08) \%$, which is satisfactory for the energy measurement of electromagnetic showers at the linear collider experiment. And despite no correction for the response curve of the MPPC yet, linearity of the energy measurement is found to be less than $4 \%$. This proves the enhancement of the effective number of pixels due to the short recovery time of the 1600 pixel MPPC. 

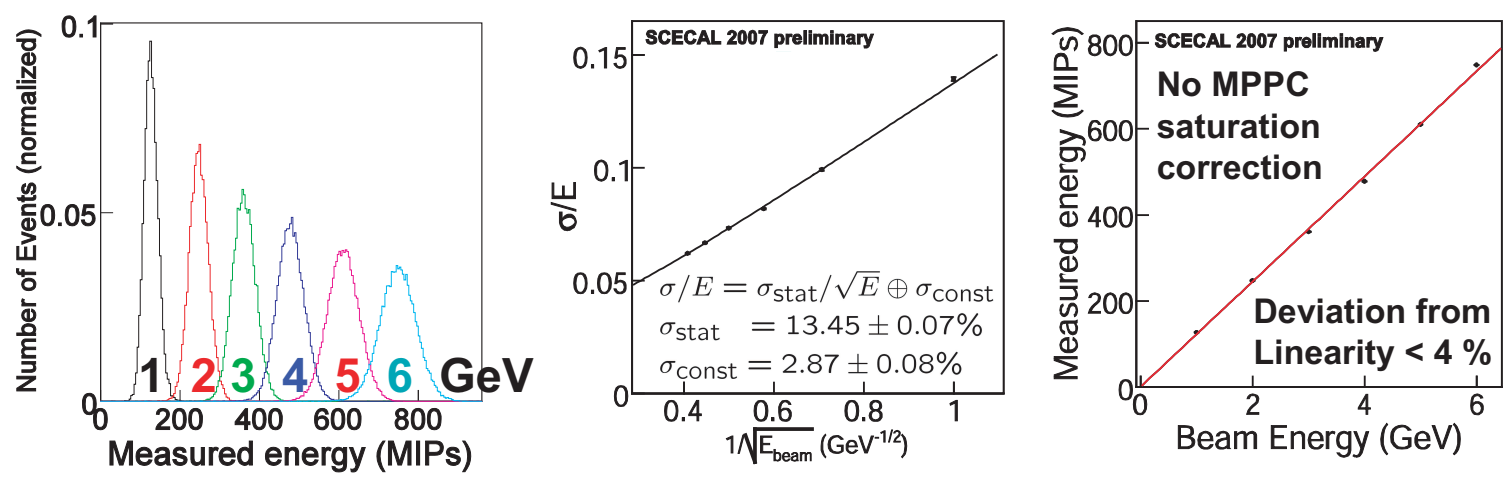

Figure 10: Energy spectra of the positrons with different momenta (left), energy resolution (middle) and linearity (right) of the GLD electromagnetic calorimeter test module.

\section{Summary}

We are developing the 1600 pixel MPPC for the GLD calorimeter readout collaborating with Hamamatsu Photonics. As a result of the study and development performed so far, the latest 1600 pixel sample shows large gain $\left(>10^{5}\right)$, low dark noise rate $(\sim 100 \mathrm{kHz})$ and cross-talk probability $(\sim 10 \%)$, and good photon detection efficiency $(\sim 17 \%)$, which are almost satisfactory for the requirements of the GLD calorimeter. The device-by-device variation of the gain and noise rate is also acceptably small. It is also found that the recovery time of the 1600 pixel MPPC is relatively short ( $\sim 4 \mathrm{~ns}$ ), which enhances the effective number of pixels for long light pulses. In total we conclude that the latest 1600 pixel MPPC is feasible for the GLD calorimeter readout, however further study is necessary to confirm radiation hardness, long-term stability and so on. Further improvements of package design, temperature dependence of breakdown voltage, and dynamic range are desired and currently ongoing.

\section{References}

[1] GLD Detector Outline Document, GLD collaboration http://ilcphys.kek.jp/gld/documents/dod/glddod.pdf

[2] Tamaki Yoshioka for the GLD Collaboration, "Particle Flow Algorithm for GLD", proceedings of the 2005 International Linear Collider Physics and Detector Workshop and 2nd ILC Accelerator Workshop, Snowmass, Colorado, 14-27 Aug 2005.

[3] Hamamatsu Photonics K.K. http://jp.hamamatsu.com/products/sensor-ssd/4010/index_en.html

[4] Masashi Yokoyama for the KEKDTP photon sensor group, "Development of Multi-Pixel Photon Counters". 2006 IEEE NSS conference record, N30-108

[5] Satoru Uozumi for the KEKDTP photon sensor group, "Development and Study of the Multi Pixel Photon Counter", proceedings for the 10th Vienna Conference on Instrumentation, Vienna, 19-24 Feb 2007.

[6] Daniel Jeans for the GLD calorimeter group, "Scintillator-tungsten ECAL testbeam", 2007 International Linear Collider Workshop, DESY, May 30 - June 32007. 